\title{
The Chinese Medicine, Shezhi Huangling Decoction, Inhibits the Growth and Metastasis of Glioma Cells via the Regulation of miR-1298-5p/TGIFI Axis
}

This article was published in the following Dove Press journal: Cancer Management and Research

\author{
Xiaoqian Liu' \\ Jianfeng Ju' \\ Qun Liu' \\ Zongmin Zhu' \\ Chunxia Liu ${ }^{2}$ \\ 'Department of Pharmacy, Affiliated \\ Hospital of Shandong University of \\ Traditional Chinese Medicine, Jinan \\ 2500II, Shandong, People's Republic of \\ China; ${ }^{2}$ Department of Pharmacy, \\ Caoxian People's Hospital of Heze City, \\ Heze 274400, Shandong, People's \\ Republic of China
}

Purpose: In recent years, traditional Chinese medicine has achieved good results in treating gliomas. This research aimed to reveal the effect of Shezhi Huangling decoction (SD) on glioma cell process.

Methods: U87 and U251 cells were treated with different concentrations $(10,30$ and $50 \mu \mathrm{g} / \mathrm{mL})$ of SD or transfected with miR-1298-5p mimic, inhibitor and siRNA targeting TGIF1. Cell proliferation, migration, invasion and apoptosis were detected. The expression of miR-1298-5p was measured by qRT-PCR, while TGIF1 expression was examined by immunohistochemical analysis and Western blot.

Results: SD treatment inhibited the proliferation, migration and invasion of glioma cells and induced the apoptosis. In addition, SD treatment induced the expression of miR-1298-5p in glioma cells. The low expression of miR-1298-5p was examined in glioma tissues and was significantly related to the high histological grade of glioma patients and predicted a poor prognosis. MiR-1298-5p directly targeted the 3'-UTR of transforming growth factor $\beta$ induced factor 1 (TGIF1) and reduced TGIF1 protein expression. MiR-1298-5p restricted the proliferation, migration and invasion of glioma cells and induced cell apoptosis by targeting TGIF1. Conclusion: Our data reveal that SD acts as a cancer-inhibiting agent in glioma via miR1298-5p/TGIF1 axis, suggesting a potential therapeutic application of SD in glioma.

Keywords: glioma, Chinese medicine, Shezhi Huangling decoction, miR-1298-5p, TGIF1

\section{Introduction}

Glioma is the most common intracranial tumor accounting for $46 \%$ of brain tumors. ${ }^{1}$ And it is one of the most lethal and rapidly progressing malignancies equivalent to $1-3 \%$ of malignant tumors detected worldwide. ${ }^{2}$ It is difficult for glioma to undergo surgical resection, due to the malignant and infiltrative growth, and the locations where it frequently occurs are important structures of brain. ${ }^{3}$ The 5 -year survival rate of malignant glioma patients treated by surgery alone is $<25 \%$. ${ }^{4}$ Reasonable postoperative radiotherapy, chemotherapy and other comprehensive treatments can control tumor growth and delay recurrence, but glioma cells are not sensitive to radiation. ${ }^{5}$ Chemotherapy drugs are difficult to penetrate the bloodbrain barrier, and there are many complications. ${ }^{6}$ There is still a lack of satisfactory treatment.

In recent years, traditional Chinese medicine has achieved good results in treating gliomas. ${ }^{7}$ One study has reported the benefits of SD and the benefits of a combination of Shezhi Huangling decoction (SD) and carmustine (BCUN) in the
Department of Pharmacy, Affiliated

Hospital of Shandong University of Traditional Chinese Medicine, No. 16369, Jingshi Road, Jinan 2500 I I, Shandong, People's Republic of China

Email yxbgongyou@163.com 
treatment of glioma compared to BCUN alone. ${ }^{8}$ Compared with BCUN alone, the combination therapy of SD and BCUN significantly improves the effectiveness of medication, the patient's functional status and survival rate. ${ }^{8}$ However, the role of SD on glioma cell function is still unknown.

MicroRNAs (miRNAs) are endogenous, small noncoding RNAs that are capable to regulate genes expression at post-transcriptional level by binding to the $3^{\prime}$-untranslated regions (3'-UTRs) of its targeted messenger RNA (mRNA). ${ }^{9}$ The interaction of miRNA-mRNA leads to mRNA degradation and translational inhibition, and miRNAs have an inhibitory effect on target gene expression. Increased evidence indicates that various miRNAs are deregulated in many types of cancers, contributing to cancer initiation and progression. ${ }^{10-12}$ Moreover, the significance of miRNAs in gliomagenesis has been reported in recent years. ${ }^{13}$ Especially, miR-1298 is identified to down-regulate expression in neuroglioma. ${ }^{14}$ Subsequently, it is also found to be down-regulated in gastric cancer and associated with lymph node metastasis and TNM stage. ${ }^{15}$ However, the specific function and molecular mechanism of miR-1298 in glioma remain unclear. In the present study, we found that SD treatment could regulate the biological function of glioma cells by affecting the expression of miR-1298-5p.

Transforming growth factor $\beta$ induced factor 1 (TGIF1), also known as TG interacting factor 1 , is a member of the three amino acid loop extension subfamily of homeodomain proteins. It is reported to repress the transcription of target genes by binding directly to DNA or binding to the retinoid $\mathrm{X}$ receptor. ${ }^{16}$ Subsequently, TGIF1 is identified as a Smadbinding protein and a transcriptional repressor involved in the TGF- $\beta$ signaling pathway. ${ }^{17}$ In humans, the TGIF1 gene is located on chromosome 18p11.3 which is the most common mutation in patients with HPE, a severe brain and craniofacial malformation associated with mental retardation, and is the part of routine genetic evaluation of HPE patients. ${ }^{18}$ Previous studies also suggest that TGIF1 play an important role in the progression of several types of cancers, including colorectal cancer, ${ }^{19}$ lung cancer, ${ }^{20}$ breast cancer ${ }^{21}$ and liver cancer. ${ }^{22}$ Especially, Shaw et al find that TGIF1 is differentially expressed in oligodendroglial tumors with $1 \mathrm{p} / 19 \mathrm{q}$ loss. ${ }^{23}$

In this study, we mainly explored the functions of SD in glioma cells. SD treatment inhibited the proliferation, migration and invasion of glioma cells, and induced the apoptosis. In addition, we found that SD treatment induced the expression of miR-1298-5p in glioma cells. Moreover, the interaction of miR-1298-5p and TGIF1 in glioma cells was identified. We demonstrated that miR-1298-5p restricted the proliferation, migration and invasion of glioma cells, and induced cell apoptosis by targeting TGIF1. In general, these findings highlighted the therapeutic potential of SD for glioma treatment.

\section{Materials and Methods Preparation of SD}

SD was composed of Hedyotis diffusa Willd. (20 g), Scutellaria barbata D. Don (15 g), Huang qi (40 g), Poria cocos (Schw) Wolf. (20 g), Atractylodes macrocephala Koidz. (18 g), Angelica sinensis (Oliv.) Diels (10 g), Rheum officinale Baill. (6 g), Kudzuvine Root (10 g). The total weight of the dried herbs was $139 \mathrm{~g}$. The herbs were blended into double-distilled water for $1 \mathrm{~h}$, then heated at $100^{\circ} \mathrm{C}$ for $2 \mathrm{~h}$, after which the residue was boiled for $2 \mathrm{~h}$ with distilled water. The extracts were subsequently diluted to $0.1 \mathrm{~g}$ herb $/ \mathrm{mL}$ and filtered with a $0.2 \mu \mathrm{m}$ filter. All medicinal plants used to prepare formulae were provided by Affiliated Hospital of Shandong University of Traditional Chinese Medicine.

\section{Clinical Samples Collection}

Thirty-eight glioblastoma tissue specimens and adjacent normal tissue specimens were collected from Affiliated Hospital of Shandong University of Traditional Chinese Medicine undergoing surgical operations. The tissue samples were immediately frozen in liquid nitrogen and stored in a $-80^{\circ} \mathrm{C}$ refrigerator. All samples were from patients who were diagnosed with glioblastoma at Affiliated Hospital of Shandong University of Traditional Chinese Medicine, had not received any other treatment except for surgery and signed the written informed consent. The experiment was approved by the Ethics Committee in Affiliated Hospital of Shandong University of Traditional Chinese Medicine.

\section{Cell Culture, Treatment and Transfection}

The human glioma cell lines (U87 and U251) were purchased from the Cell Bank of the Chinese Academy of Sciences (Shanghai, China) and were cultured as previously described. ${ }^{24}$ For SD treatment, the cells were incubated in a medium containing a different concentration of SD reagent. Phosphate Buffered Saline (PBS) was used as negative control (NC). The miR-1298-5p mimic, inhibitor and siRNA-TGIF1 were synthesized from Ruibo (Guangzhou, China). The oligonucleotide sequence used 
were as follows: miR-1298-5p mimic, 5'-TTCATTC GGCTGTCCAGATGTA-3'; inhibitor, 5'-TACATCTGGA CAGCCGAATGAA-3'; siRNA-TGIF1, 5'-CCGATCAAG CCTGACTTCT-3'. Lipofectamine 2000 (Invitrogen, Carlsbad, CA) was used to transfer them into U87 and U251 cells.

\section{Quantitative Reverse-Transcription Polymerase Chain Reaction}

Total RNAs were isolated from tissues and cells by using TRIzol reagent (Invitrogen). For reverse transcription, miRNAs were reverse transcribed to cDNAs using TaqMan Advanced miRNA cDNA Synthesis Kit (Applied Biosystems, Foster City, CA, USA) and mRNAs were reverse transcribed to cDNAs using First-Strand cDNA Synthesis Kit (Promega Corporation, Madison, WI). Quantitative reverse-transcription polymerase chain reaction (qRT-PCR) was performed using SYBR Green Master Mix Kit (Takara, Otsu, Japan) to amplification for gene expression. Relative miR-1298-5p and TGIF1 expression were obtained by normalizing with U6 and GAPDH, respectively, and quantified with the $2^{-\Delta \Delta \mathrm{Ct}}$ method. The primers used were as follows: miR-1298 forward, 5'-TCATTCGG CTGTCCAGA-3' and reverse, 5'- GAACATGTCTGCG TATCTC-3'; U6 forward, 5'-CTCGCTTCGGCAGCA CA-3' and reverse, 5'-AACGCTTCACGAATTTGCGT-3'; TGIF1 forward, 5'-GGATTGGCTGTATGAGCACCGT-3' and reverse, 5'-GCCATCCTTTCTCAGCATGTCAG-3'; GAPDH forward, 5'-ATGAGCTCCACTCTGCTCAATGT CACGG-3' and reverse, 5'-GCAAGCTTCTCTACCAAG AATGAAAGAGCAT-3'.

\section{Measurement of Cell Proliferation}

CCK8 and colony formation assay were performed to determine cell proliferation. For CCK 8 assay, $5 \times 103$ cells were seeded onto each well in the 96-well plates and cultured overnight. For SD treatment, the cells were incubated in a medium containing a different concentration of SD reagent for 12 h, 24 h, $36 \mathrm{~h}$ and $48 \mathrm{~h}$. For transfection, the cells were transfected by miR-1298-5p mimic, inhibitor or siRNA-TGIF1, and continue to culture for $0 \mathrm{~h}$, $24 \mathrm{~h}, 48 \mathrm{~h}$ and $72 \mathrm{~h}$. Then, culture medium was removed, $100 \mu \mathrm{L}$ of fresh medium containing $10 \mu \mathrm{L}$ CCK8 solution (Beyotime Biotechnology, Shanghai, China) was added to each well and incubated at $37^{\circ} \mathrm{C}$ for $2 \mathrm{~h}$. The $\mathrm{OD}$ value at $450 \mathrm{~nm}$ was measured using a microplate reader. For colony formation assay, 500 cells were seeded into 6-well plate. After two weeks, the cells grew to visible colonies and were fixed with methanol for $30 \mathrm{~min}$, stained with $0.1 \%$ crystal violet for $1 \mathrm{~h}$. The colonies were counted and imaged.

\section{Transwell Assay}

Transwell chamber (24-well) was performed to examine cell migration and invasion; $24 \mathrm{~h}$ after SD treatment or transfection, cells were resuspended in serum-free medium and adjusted to $1 \times 105$ cells $/ \mathrm{mL}$. Then, $200 \mu \mathrm{L}$ of cell suspension was put into the upper chamber, and 500 $\mu \mathrm{L}$ of medium containing $10 \%$ FBS was added into the lower chamber to induce cells to migrate or invade through the $8-\mu \mathrm{m}$ pore-size membrane. In addition, the cells were put in the upper chamber with a thin layer Matrigel matrix (BD Biosciences, Franklin Lakes, NJ) for invasion assay. The other experimental steps are the same as the migration experiment; $24 \mathrm{~h}$ later, cells that migrated or invaded to the lower surface of the membrane were fixed with $4 \%$ paraformaldehyde and stained with $0.1 \%$ crystal violet. Finally, the number of moving cells was counted in five randomly chosen fields using a microscope.

\section{Flow Cytometry Analysis}

Then, $48 \mathrm{~h}$ after SD treatment or transfection, cells were resuspended to $1-5 \times 10^{6} / \mathrm{mL}$ by Annexin V binding buffer. Cells were double stained by FITC Annexin V Apoptosis Detection Kit (BD Biosciences, Franklin Lakes, NJ, USA) and detected by flow cytometry. Statistical analysis was performed using Flowjo software (Tree Star, Ashland, OR, USA).

\section{Luciferase Activity Assay}

The wild binding sites between miR-1298-5p and TGIF1 (TGIF1-WT) and mutated binding sites (TGIF1-Mut) were synthesized and cloned into psiCHECK-2 Vector (Promega, Madison, WI). The psiCHECK-2-TGIF1-WT or psiCHECK2-TGIF1-Mut vector (Promega) was transfected into HEK293T cells with miR-1298-3p mimics or negative control miRNA (miR-NC); $48 \mathrm{~h}$ after co-transfection, luciferase activity was determined using a Dual-Luciferase Reporter Assay System (Promega).

\section{Western Blot}

Proteins from tissues and cells were lysed in RIPA buffer containing protease and phosphatase inhibitors (Roche, Basel, Switzerland) and were harvested by centrifugation 
with $12,000 \mathrm{rpm}$ for $10 \mathrm{~min}$ at $4^{\circ} \mathrm{C}$. BCA Protein Assay Kit (Tiangen, Beijing, China) was used to quantify the protein concentrations. Equal amounts of proteins $(15 \mathrm{mg}$ ) were separated through 10\% SDS-PAGE gel and then transferred onto PVDF membranes. Next, the membranes were incubated with anti-TGIF1 (1:1000, ab52955, Abcam, Cambridge, MA) at $4{ }^{\circ} \mathrm{C}$ overnight after blocked with $5 \%$ non-fat milk for $1 \mathrm{~h}$ at normal temperature and then subsequently incubated with HRP conjugated secondary antibody at room temperature for $2 \mathrm{~h}$. Immunoreactive bands were developed using Enhanced chemiluminescence kit (Amersham, Little Chalfont, UK).

\section{Immunohistochemical Analysis}

The glioma or adjacent tissues were fixed in formalin for 24 $\mathrm{h}$ and then embedded in paraffin. The paraffin sample was cut into $4 \mu \mathrm{m}$ slides, and the slides were deparaffinized in an environmentally friendly clearing agent (Hongci., Shanghai, China) and rehydrated in alcohol. Sodium citrate buffer $(\mathrm{pH}$ 6.0) was used for antigen retrieval. After blocked with 5\% sheep serum at room temperature for $1 \mathrm{~h}$, slides were incubated with anti-TGIF (1:100) for $1 \mathrm{~h}$ at room temperature, and then was incubated with secondary antibody (160101405L, Maixin., Shanghai, China). The enhanced DAB chromogenic kit $(1,705,252,031$, Maixin., Shanghai, China) is used for color development. After staining once with hematoxylin, it was dehydrated with a gradient ethanol and fixed with neutral resin. And the slides were observed and imaged under a microscope.

The immunostaining score was calculated by multiplying positive staining cell ratio $(\mathrm{R})$ and staining intensity score (S) which were given by two pathologists blinded to the clinical parameters. $\mathrm{R}$ was divided into four grades: $0(<5 \%$, negative), 1 (5-25\%, sporadic), 2 ( $25-50 \%$, focal), 3 ( $>51 \%$, diffuse), $\mathrm{S}$ was also divided into four grades: 0 (negative), 1 (weak), 2 (moderate), 3 (strong). Finally, $0-4$ was considered to be low staining, and 6-9 was considered to be high staining.

\section{Statistical Analysis}

All results were presented as the mean $\pm \mathrm{SD}$ and conducted using SPSS 22.0 (SPSS, IBM, Beijing, China) and GraphPad Prism 6 (GraphPad, San Diego, CA). The difference was calculated by Student $t$-test or one-way analysis of variance. The survival curves were generated using the Kaplan-Meier method followed by Log rank test. It was defined as significant differences at $\mathrm{P}<0.05$.

\section{Results}

\section{SD Treatment Reduces the Malignant Phenotype of Glioma Cells in vitro}

To reveal the effect of SD on the glioma cell process, U87 and U251 cells were treated with different concentrations of $\mathrm{SD}$, and cell proliferation, migration, invasion and apoptosis were detected. As shown in Figure 1A, SD treatment $(10,30$ and $50 \mu \mathrm{g} / \mathrm{mL})$ significantly inhibited the proliferation of U87 and U251 cells, the number of viable cells decreases as the SD concentration and treatment time increases $(P<0.05)$. Based on the data of CCK 8 assay, $30 \mu \mathrm{g} / \mathrm{mL}$ of $\mathrm{SD}$ reagent was used to the subsequent experiments. Furthermore, the results of transwell assay have shown that the migration and invasion of U87 and U251 cells were obviously inhibited following $\mathrm{SD}$ treatment (Figure $1 \mathrm{~B}$ and $\mathrm{C} ; \mathrm{P}<0.05$ ). Additional evidences suggested that SD treatment induced the apoptosis of U87 and U251 cells (Figure 1D). The apoptosis percentage increased from $4.39 \pm 0.52 \%(3.82 \pm 0.12 \%)$ to $14.26 \pm 0.74 \%(10.31 \pm 0.57 \%)$ of $\mathrm{U} 87$ (U251) cells (P $<0.05)$.

\section{SD Treatment Induces the Expression of miR- I298-5p}

U87 and U251 cells were treated with different concentrations of SD, and we found that miR-1298-5p level was increased following the treatment of SD (Figure 2A). These results made us to speculate that SD may regulate the biological function of glioma cells by affecting the expression of miR-1298-5p.

\section{Downregulated miR-I298-5p Is} a Prognostic Factor for Glioma Patients

To identify the roles of miR-1298-5p in glioma, the expression pattern of it was examined in 38 cases glioblastoma tissues and 38 cases paired adjacent normal tissues by qRT-PCR. The expression of miR-1298-5p was significantly down-regulated in glioblastoma samples compared into normal tissues (Figure 2B; $\mathrm{P}<0.05$ ). In order to analyze the correlation between miR-1298-5p expression and clinical characteristics of glioblastoma patients, all glioblastoma tissues samples were divided into two groups based on the miR-1298-5p expression. As shown in Table 1, low expression of miR-1298-5p was significantly related to the high histological grade of the glioblastoma patients (Table $1 ; \mathrm{P}=0.016685$ ). In 

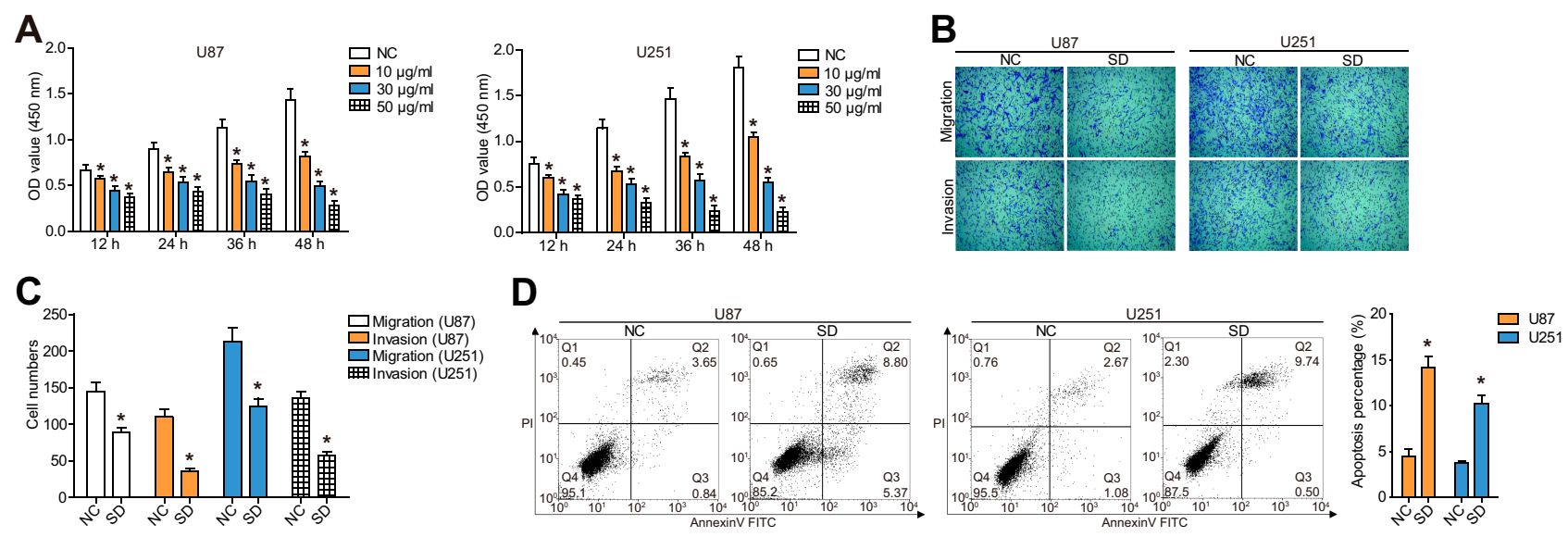

Figure I SD treatment reduces the malignant phenotype of glioma cells in vitro.

Notes: (A) The proliferation of U87 and U25I cells at different concentrations of SD treatment ( 10,30 and $50 \mu g / \mathrm{mL})$ and at different treatment times was examined by CCK-8 assay. (B and C) Cell migration and invasion potential of U87 and U25I cells at $30 \mu g / \mathrm{mL}$ SD for $24 \mathrm{~h}$ was measured by transwell assay. (D) Cell apoptosis of U87 and $\mathrm{U} 25 \mathrm{I}$ cells at $30 \mu \mathrm{g} / \mathrm{mL}$ SD for $48 \mathrm{~h}$ was detected by Flow cytometry analysis. $* \mathrm{P}<0.05$.

Abbreviations: NC, negative control; SD, Shezhi Huangling decoction.

addition, the overall survival of patients with high miR1298-5p expression was higher than that of patients with low miR-1298-5p expression (Figure $2 \mathrm{C} ; \mathrm{P}=0.0278$ ). According to these results, we inferred that miR-1298-5p is a crucial prognostic factor for glioblastoma.

\section{miR-1298-5p Reduces the Malignant Phenotype of Glioma Cells in vitro}

To further investigate the function of miR-1298-5p, U87 and U251 cells were transfected with miR-1298-5p mimic and the changes of their proliferation, migration, invasion and apoptosis were detected. RT-qPCR detection confirmed that miR-1298-5p expression was up-regulated in miR-1298$5 \mathrm{p}$ mimic-transfected cells (Figure 2D; $\mathrm{P}<0.05$ ). The CCK8 assay revealed that up-regulated miR-1298-5p significantly decreased the proliferation of U87 and U251 cells (Figure 2E; $\mathrm{P}<0.05$ ). The results of transwell assay also indicated that up-regulated miR-1298-5p repressed cell migration and invasion in U87 and U251 cells (Figure 2F; $\mathrm{P}<0.01)$. Moreover, over-expression of miR-1298-5p induced the apoptosis of U87 and U251 cells (Figure 2G; $\mathrm{P}<0.05)$. Taken together, these results suggest that miR$1298-5 p$ reduces the malignant phenotype of glioma cells in vitro.

\section{SD Treatment Regulates the Biological Function of Glioma Cells by Affecting the Expression of miR-1298-5p}

In order to confirm whether SD treatment regulated the glioma cell process by affecting miR-1298-5p expression,
U87 and U251 cells were subjected to miR-1298-5p inhibitor (miR-inhibitor) and SD treatment at the same time. As shown in Figure 3A, the transfection of miR-inhibitor significantly decreased the miR-1298-5p level, while SD treatment complemented the role of miR-inhibitor. Moreover, SD treatment significantly inhibited the proliferation, migration and invasion of U87 and U251 cells, while transfection of miR-inhibitor complemented the affection of SD treatment (Figure 3B and $\mathrm{C} ; \mathrm{P}<0.05$ ). In addition, the transfection of miR-inhibitor hindered the induction of apoptosis by SD treatment (Figure 3D). These results revealed that SD treatment regulates the biological function of glioma cells by affecting the expression of miR-1298-5p.

\section{TGIFI Is a Direct Target Gene of miR-I298-5p}

Furthermore, TGIF1 was predicted as a potential target gene of miR-1298-5p in TragetScan database (http:// www.targetscan.org/vert 72/) to uncover the downstream molecular mechanism of miR-1298-5p (Figure 4A). The 3' UTR of TGIF1 contained a complementary site for the seed region of miR-1298-5p. The results of luciferase reporter assay showed that miR-1298-5p over-expression declined the luciferase activity of TGIF1-WT, but had no obvious effect on the luciferase activity of TGIF1-Mut (Figure 4B; $\mathrm{P}<0.05$ ), suggesting that miR-1298-5p directly targeted TGIF1 $3^{\prime}$ UTR via the predicted seed region. And the protein expression of TGIF1 was significantly decreased by miR-1298-5p over-expression in U87 

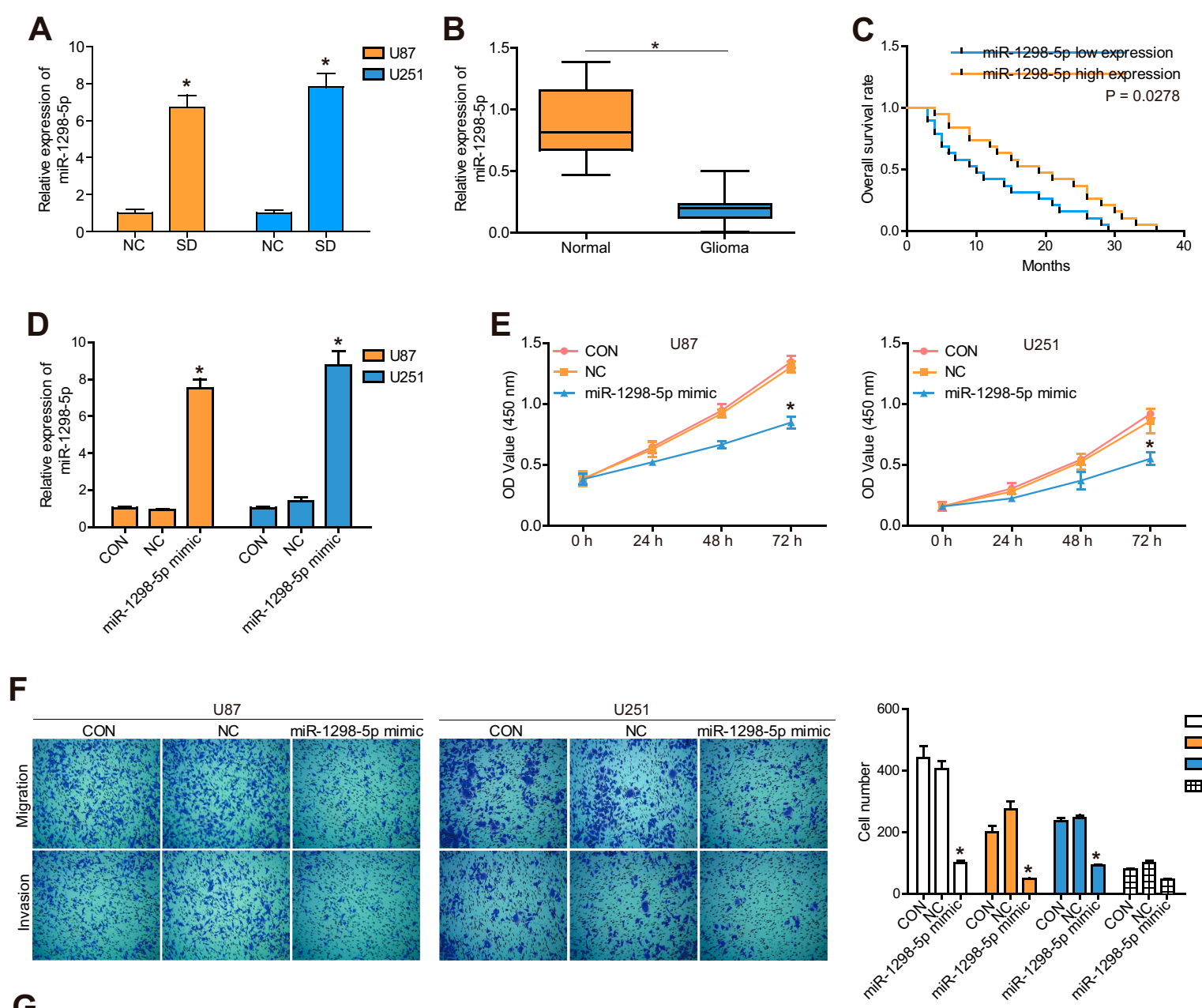

G
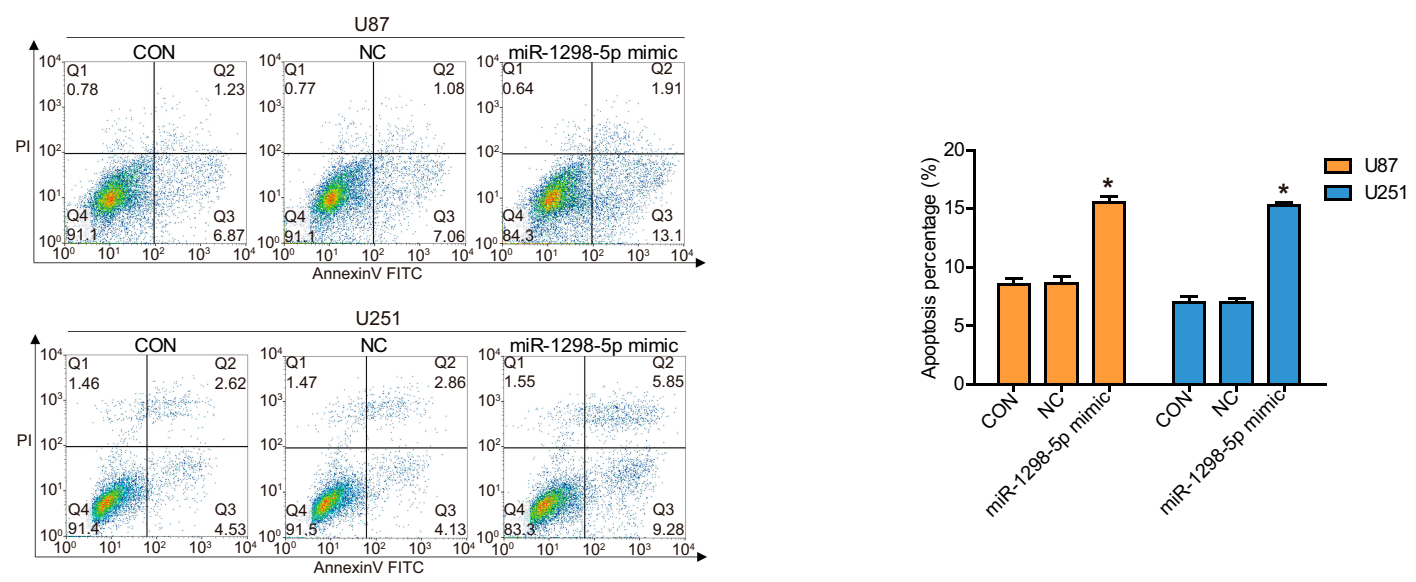

Figure 2 Downregulated miR-1298-5p is a prognostic factor for glioma patients.

Notes: (A) RT-qPCR analysis of miR-1298-5p expression levels in SD-treated cells. (B) RT-qPCR analysis of miR-1298-5p expression levels in glioblastoma tissues and paired adjacent normal tissues. (C) Kaplan-Meier analysis of glioblastoma patients with different miR-1298-5p expression. (D) The expression of miR-1298-5p was detected by RTqPCR in U87 and U25I cells that were transfected with miR-I298-5p mimic. (E) Cell proliferation of U87 and U25I cells transfected with miR-I298-5p mimic was examined by CCK-8 assay. (F) Cell migration and invasion potential of U87 and U25I cells transfected with miR-I298-5p mimic was measured by transwell assay. (G) Cell apoptosis of U87 and U25I cells transfected with miR-1298-5p mimic was detected by Flow cytometry analysis. *P $<0.05$.

Abbreviations: SD, Shezhi Huangling decoction; CON, control; NC, negative control.

and $\mathrm{U} 251$ cells (Figure $4 \mathrm{C}$ and $\mathrm{D} ; \mathrm{P}<0.05$ ). These results indicate that miR-1298-5p directly targets TGIF1 3' UTR and regulates TGIF1 expression in glioma cells.

\section{TGIFI Is Up-Regulated in Glioma Tissues}

The obviously high expression of TGIF1 was identified in glioma tissues detected by GEPIA analysis, 
Table I Correlation Between miR-1298-5p and TGIFI Expression and Clinical Features of Glioma Patients

\begin{tabular}{|c|c|c|c|c|c|c|c|}
\hline \multirow[t]{2}{*}{ Parameters } & \multirow[t]{2}{*}{ Cases, $\mathbf{N}=\mathbf{3 8}$} & \multicolumn{2}{|c|}{ miR-I 298-5p Expression } & \multirow[t]{2}{*}{ P-value } & \multicolumn{2}{|c|}{ TGIF I Expression } & \multirow[t]{2}{*}{ P-value } \\
\hline & & Low $\mathbf{N}=19$ & High, $\mathbf{N}=19$ & & Low, $\mathbf{N}=9$ & High, $\mathbf{N}=29$ & \\
\hline Age (years) & & & & 0.515830 & & & 0.840617 \\
\hline$<60$ & 18 & 8 & 10 & & 4 & 14 & \\
\hline$\geq 60$ & 20 & 11 & 9 & & 5 & 15 & \\
\hline Sex & & & & 0.641550 & & & 0.802746 \\
\hline Male & 24 & 13 & II & & 6 & 18 & \\
\hline Female & 14 & 6 & 8 & & 3 & 11 & \\
\hline WHO grade & & & & $0.016685 *$ & & & $0.018805 *$ \\
\hline $1+11$ & 13 & 3 & 10 & & 6 & 7 & \\
\hline$I I I+I V$ & 25 & 16 & 9 & & 3 & 22 & \\
\hline
\end{tabular}

Note: $* \mathrm{P}<0.05$.

immunohistochemistry and Western blot. GEPIA analyzed the TGIF1 expression profiling dataset from TGCA, including 163 cases of glioblastoma (GBM) tissues and 207 cases of normal brain tissues. These data showed that TGIF1 was significantly over-expressed in GBM tissues, compared to normal brain tissues (Figure 4E; $\mathrm{P}<0.05$ ). Secondly, the results of immunohistochemistry indicated that weak TGIF1 immunostaining occurred in the cytoplasm and nucleus of normal tissues, while strong TGIF1 immunostaining occurred in the cytoplasm and nucleus of glioblastoma tissues (Figure 4F). TGIF1 was highly expressed in glioblastoma tissues $(29 / 38,76.3 \%$ ), and the high expression of TGIF1 was significantly correlated to the high histological grade of the glioblastoma patients (Table 2). Furthermore, significantly higher protein expression of TGIF1 was detected in glioblastoma tissues compared to normal tissues by Western blot (Figure 4G and H; $\mathrm{P}<0.05$ ). In addition, the relative expression of miR-1298-5p was negatively correlated with the expression of TGIF1 in glioblastoma tissues (Figure 4I; $\mathrm{P}<0.001)$. We also found that the treatment of SD inhibited the protein expression of TGIF1 in U87 and U251 cells (Figure 4J and K), which was contrary to the effect of SD on miR-1298-5p levels. We speculate that SD treatment impede the expression of TGIF1 by inducing miR-1298-5p expression.

\section{The Effects of TGIFI Knockdown on the Proliferation, Migration, Invasion, EMT Progress and Apoptosis of Glioma Cells in vitro}

U87 and U251 cell lines were transfected with siRNATGIF1, and down-regulated TGIF1 expression was found in transfected U87 and U251 cells (Figure 4L and M). The results of CCK8 and colony formation assays indicated that knockdown of TGIF1 significantly restricted the proliferation of U87 and U251 cells (Figure 5A and B; P < 0.05). Results of transwell assay also showed the repression effects of TGIF1 knockdown on migration and invasion of U87 and U251 cells (Figure 5C; $\mathrm{P}<0.05$ ). Furthermore, flow cytometry analysis found that the cell apoptosis was observably accelerated by TGIF1 knockdown (Figure 5D; $\mathrm{P}<0.05$ ).

\section{Discussion}

Intervening the growth and development of cancer with naturally occurring herbs and phytochemicals is essential to improving the treatment of cancer and reducing mortality. Chinese herbal medicine has been widely used in the treatment of cancer in East Asian and has been regarded as new sources of drug discovery library. ${ }^{7}$ Some traditional Chinese medicine extracts or compounds, like Berberine, Dauricine and Fucoidan are shown to induce autophagic cell death in cancer cells, via m-TOR or Beclin1dependent. $^{25}$ In the present study, we firstly found that SD treatment inhibited the proliferation, migration and invasion of glioma cells, and induced the apoptosis. In addition, we found that SD treatment induced the expression of miR-1298-5p in glioma cells.

MiRNA dysregulation plays a crucial role in many types of cancers and their differential expression regulates multiple biological behaviors. ${ }^{26}$ MiR-1298 is identified later, so there are relatively few reports on miR-1298. Firstly, miR1298 is identified as novel miRNA that inhibits the growth of KRAS-driven cancer cells both in vitro and in vivo. ${ }^{27}$ Moreover, the expression of miR-1298 is down-regulated in 
A

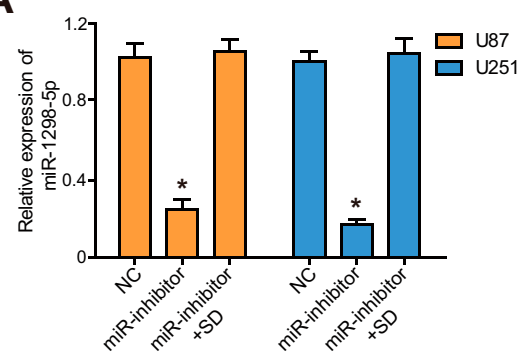

C

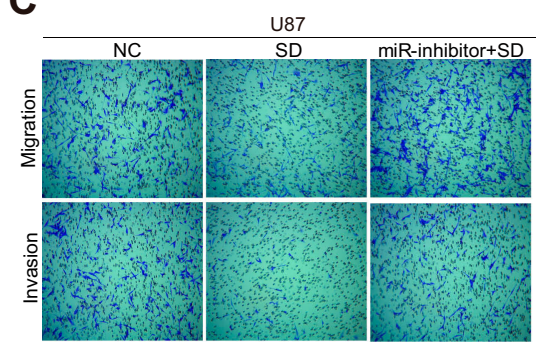

B
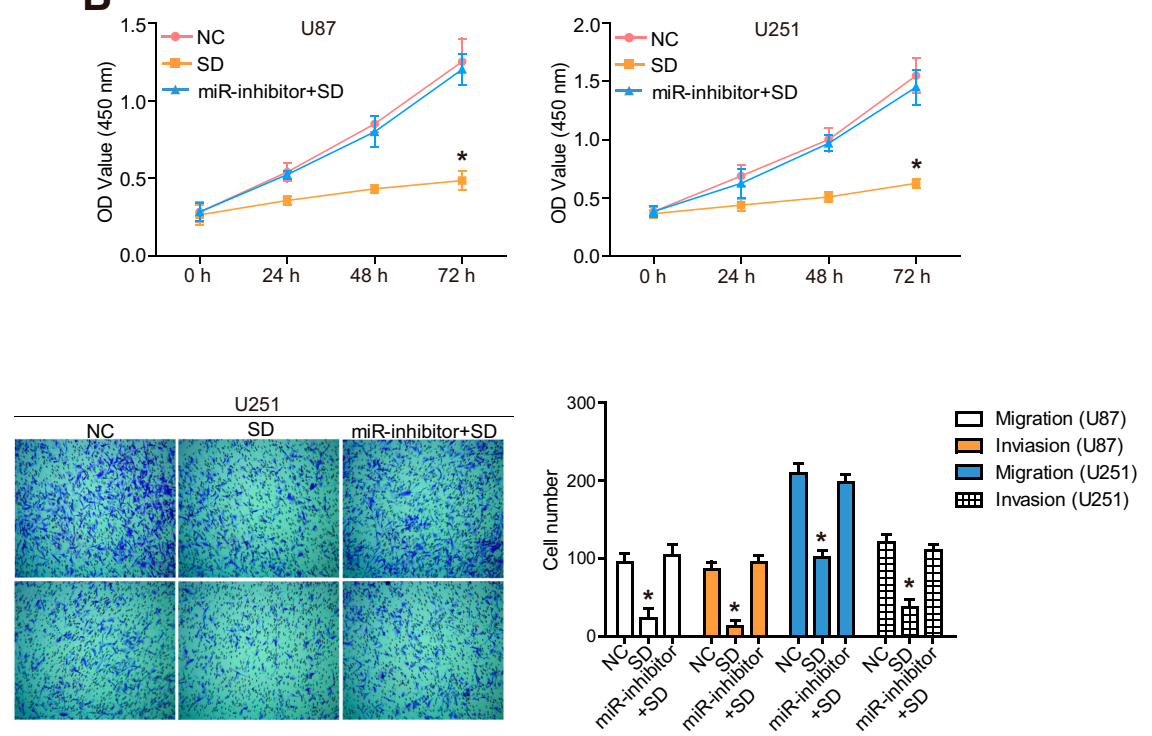

D
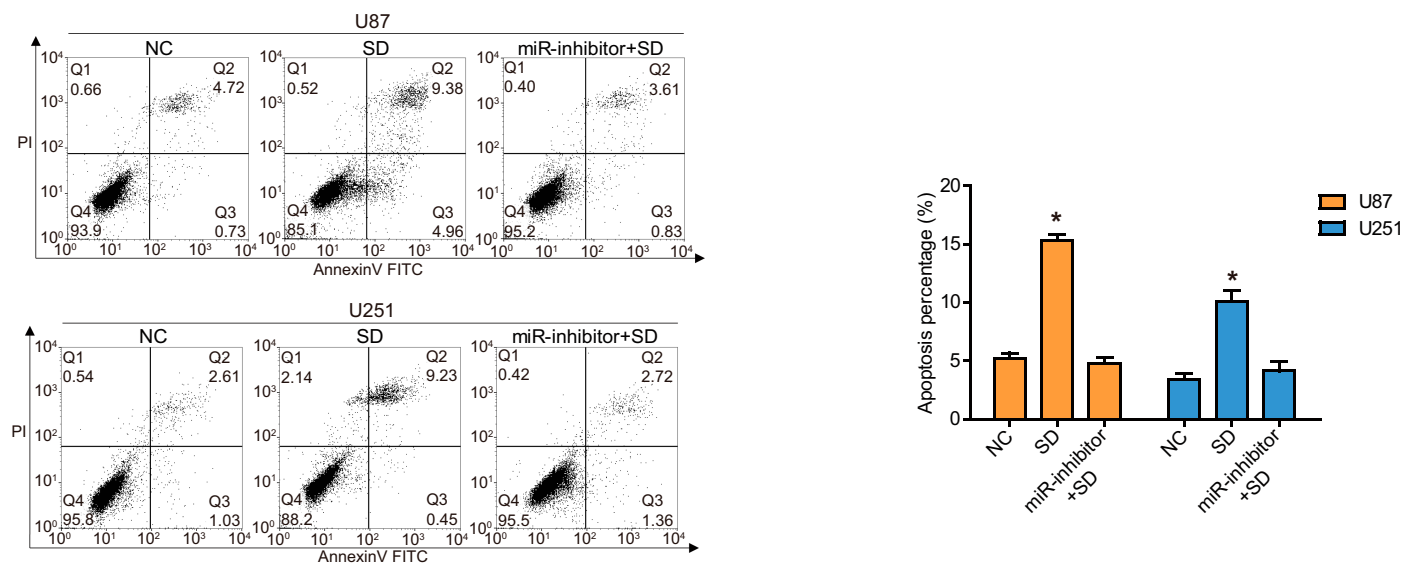

Figure 3 SD treatment regulates the biological function of glioma cells by affecting the expression of miR-1298-5p.

Notes: (A) The expression of miR-1298-5p was detected by RT-qPCR in U87 and U25I cells that were transfected with miR-1298-5p inhibitor (miR-inhibitor) and treated with SD. (B) Cell proliferation of U87 and U25I cells transfected with miR-inhibitor and treated with SD was examined by CCK-8 assay. (C) Cell migration and invasion potential of U87 and U25I cells transfected with miR-inhibitor and treated with SD was measured by transwell assay. (D) Cell apoptosis of U87 and U25I cells transfected with miR-inhibitor and treated with SD was detected by Flow cytometry analysis. $* \mathrm{P}<0.05$.

Abbreviations: NC, negative control; SD, Shezhi Huangling decoction; miR-inhibitor, miR-I298-5p inhibitor.

gastric cancer tissues and cells, and miR-1298 overexpression inhibits cell proliferation and invasion. ${ }^{15}$ Lower miR-1298 expression levels are associated with lymph node metastasis and TNM stage, and predict poor disease-free survival (DFS) and OS of patients with gastric cancer. ${ }^{15}$ Importantly, miR-1298 is identified as obviously down-regulated in neuroglioma. ${ }^{14}$ In addition, rno-miR -1298 is detected to be significantly down-regulated in rat glioma C6 cells. $^{28}$ Over-expression of rno-miR-1298 obviously inhibits the proliferation and induces apoptosis in C6 cells. ${ }^{28}$ In this study, we found that miR-1298-5p was decreased in glioblastoma tissues and the over-expression of miR-1298-5p inhibited the proliferation, migration and invasion and induced apoptosis of glioma cells. Furthermore, lower miR-1298-5p expression was related to the high histological grade of glioblastoma patients and predicted poor OS. Based on previous studies, we explored the function of miR-1298-5p in glioma cells.

Moreover, SD treatment significantly inhibited the proliferation, migration and invasion of U87 and U251 cells and induced the expression of miR-1298-5p, while transfection of miR-inhibitor complemented the affection of SD treatment. In addition, the transfection of miR-inhibitor hindered the induction of apoptosis by SD treatment. These results revealed that SD treatment regulates the biological function of glioma cells by affecting the 
A

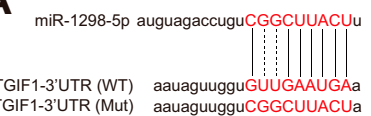

B

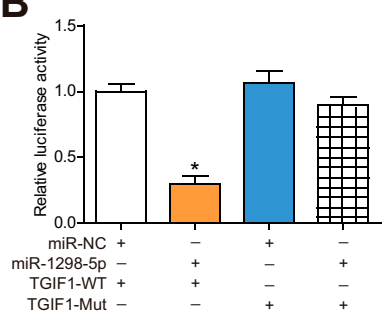

G

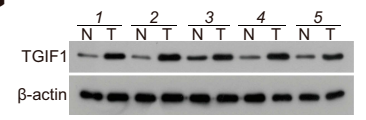

H

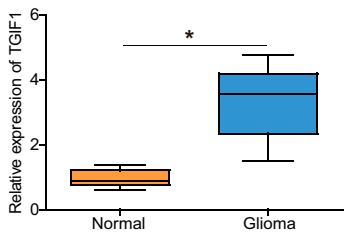

C

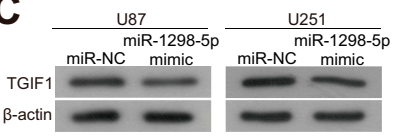

D

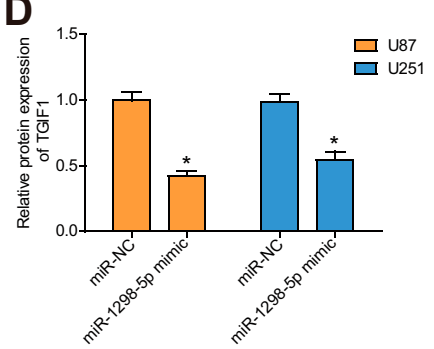

I

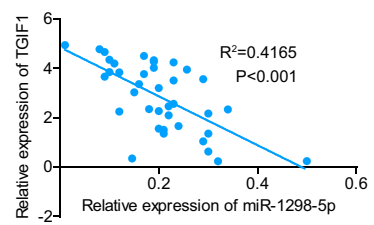

E

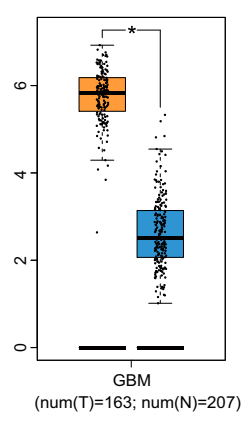

F

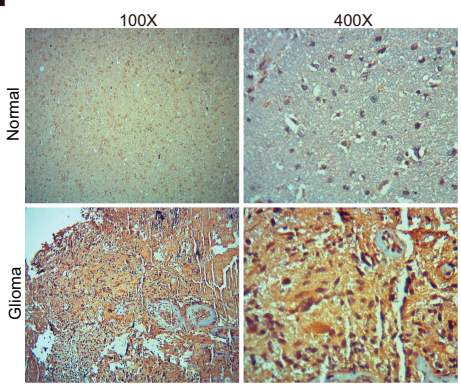

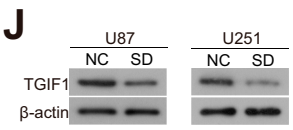

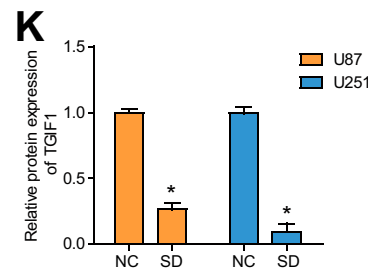

L

\section{M}

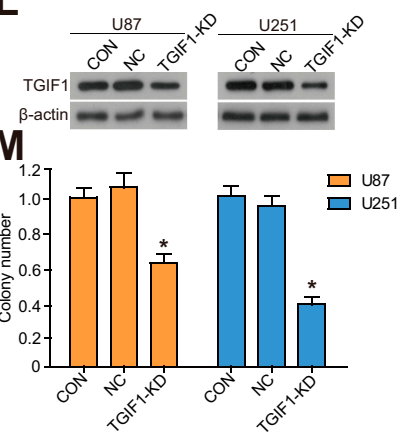

Figure 4 TGIFI is a direct target gene of miR-1298-5p.

Notes: (A) MiR-1298-5p had binding sites with the 3'-UTR of TGIFI. (B) TGIFI 3'-UTR luciferase reporter assay. (C and D) The protein expression of TGIFI of U87 and U25I cells transfected with miR-1298-5p mimic was detected by Western blot. (E) The mRNA expression of TGIFI in glioblastoma (GBM) tissues and normal brain tissues. All data came from TCGA. The protein expression of TGIFI was detected by immunohistochemical $(\mathbf{F})$ and Western blot $(\mathbf{G}$ and $\mathbf{H})$ in glioblastoma tissues and normal brain tissues. (I) The correlation analysis of miR-1298-5p and TGIFI expression in glioblastoma tissues. (J and K) The protein expression of TGIFI in SD treated cells was detected by Western blot. (L and $\mathbf{M}$ ) The protein expression of TGIFI was detected by Western blot in U87 and U25I cells that were transfected with siRNA-TGIFI (TGIFI-KD). *P $<0.05$.

Abbreviations: WT, wild type; Mut, mutant type; miR-NC, negative control miRNA; SD, Shezhi Huangling decoction; CON, control; NC, negative control; TGIFI-KD, knockdown of TGIFI.

expression of miR-1298-5p, although we have not disclosed its specific molecular regulatory mechanism.

Further, it has been reported that miR-1298 is involved in the pathogenesis of cancers through targeting some genes, such as FAK, LAMB3, ${ }^{27}$ SETD7. ${ }^{28}$ In this study, we also demonstrated that miR-1298-5p directly target TGIF1 to participate in the progression of glioma. MiR-1298-5p bound to the 3'-UTR of TGIF1 and decreased TGIF1 protein expression. Moreover, we

Table 2 TGIFI Expression in Glioblastoma Tissues Compared with Normal Brain Tissues

\begin{tabular}{|l|l|l|l|l|}
\hline \multirow{2}{*}{ Group } & \multirow{n}{*}{$\mathbf{l}$} & \multicolumn{2}{|l|}{ TGIFI Expression } & \multirow{2}{*}{ P } \\
\cline { 3 - 4 } & & Low (n\%) & High (n\%) & \\
\hline Glioblastoma & 38 & $9(23.7)$ & $29(76.3)$ & \multirow{2}{*}{$0.0024 I^{*}$} \\
Normal & 38 & $22(57.9)$ & $16(42.1)$ & \\
\hline
\end{tabular}

Note: $* \mathrm{P}<0.05$ found that TGIF1 was up-regulated in human glioblastoma tissues. Knockdown of TGIF1 inhibited cell proliferation, migration and invasion of glioma cells and induced cell apoptosis. In previous studies, TGIF1 has been found to be up-regulated in lung cancer tissues and cell lines, ${ }^{29}$ metastasis breast cancer, ${ }^{21}$ metastasis lung adenocarcinoma, $^{20}$ non-small cell lung cancer (NSCLC), ${ }^{30}$ and colon cancer. ${ }^{19}$ Moreover, downregulation of TGIF1 impairs the growth, migration and invasion of NSCLC cells (A549 and H23) ${ }^{30,31}$ and breast cancer cells (MDA-MB-231). ${ }^{21}$ In addition, one research has shown that TGIF1 expression is down-regulated in $1 p / 19 q-d e f i c i e n t \quad$ oligodendroglial. $^{23}$ Oligodendroglial $^{2}$ tumors with $1 \mathrm{p} / 19 \mathrm{q}$ loss are more likely to be chemosensitive and have longer survival. ${ }^{32}$ Thus, in line with previous studies, our funding supported the notion that TGIF1 is a tumor promoter in glioma that may be a new therapeutic target in the future. 

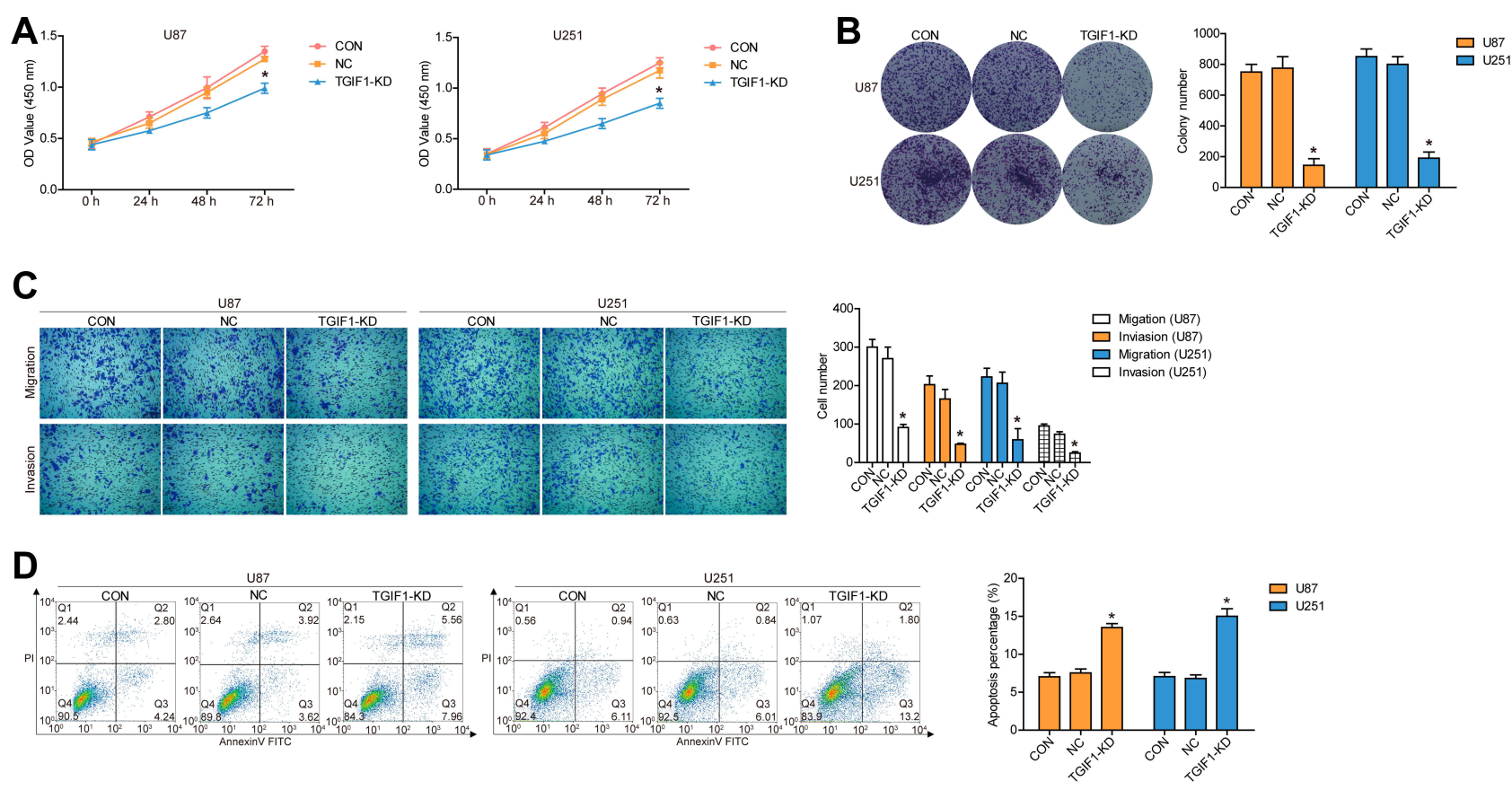

Figure $\mathbf{5}$ The effects of TGIFI knockdown on the proliferation, migration, invasion and apoptosis of glioma cells in vitro.

Notes: Cell proliferation was examined by CCK-8 assay $(\mathbf{A})$ and colony formation assay $(\mathbf{B})$. (C) Cell migration and invasion potential was measured by transwell assay. (D) Cell apoptosis was detected by Flow cytometry analysis. $* \mathrm{P}<0.05$.

Abbreviations: CON, control; NC, negative control; TGIFI-KD, knockdown of TGIFI.

\section{Conclusions}

In conclusion, we found for the first time the role of SD, the down-regulation of miR-1298-5p and up-regulation of TGIF1 in glioma. And SD acts as a cancer-inhibiting agent in glioma via miR-1298-5p/TGIF1 axis. These data suggest a potential therapeutic application of SD in glioma.

\section{Ethical Approval}

The experiment was approved by the Ethics Committee of the Affiliated Hospital of Shandong University of Traditional Chinese Medicine.

\section{Funding}

This work was supported by grants from the Science and Technology Development Plans of Traditional Chinese Medicine of Shandong Province (No. 2019-0073), and 2019 national inheritance talent training project of TCM characteristic technology (T20194828003).

\section{Disclosure}

All authors declare that they have no conflicts of interest.

\section{References}

1. Persaud D, Burns J, Trangle J, Moulik S. Disparities in brain cancer in the United States: a literature review of gliomas. Med Sci. 2017;5 (3):16. doi:10.3390/medsci5030016

2. Weller M, Wick W, Aldape K, et al. Glioma. Nat Rev Dis Primers. 2015;1(2015):15017. doi:10.1038/nrdp.2015.17

3. Hervey-Jumper SL, Berger MS. Maximizing safe resection of low- and high-grade glioma. J Neurooncol. 2016;130(2):269-282. doi:10.1007/ s11060-016-2110-4

4. Krivosheya D, Prabhu SS, Weinberg JS, Sawaya R. Technical principles in glioma surgery and preoperative considerations. J Neurooncol. 2016;130(2):243-252. doi:10.1007/s11060-016-2171-4

5. Wang J, Wang Y, He Y, et al. Radiotherapy versus radiotherapy combined with temozolomide in high-risk low-grade gliomas after surgery: study protocol for a randomized controlled clinical trial. Trials. 2019;20(1):641. doi:10.1186/s13063-019-3741-5

6. Sorensen MD, Fosmark S, Hellwege S, Beier D, Kristensen BW, Beier CP. Chemoresistance and chemotherapy targeting stem-like cells in malignant glioma. Adv Exp Med Biol. 2015;853:111-138.

7. Zhong C, Zhang YF, Huang JH, et al. The Chinese medicine, Jianpi Huayu decoction, inhibits the epithelial mesenchymal transition via the regulation of the Smad3/Smad7 cascade. Am J Transl Res. 2017;9 (6):2694.

8. Jiang R, Dong W, Yan Z, Wang D, Li X. Clinical and experimental study of Shezhi Huangling decoction on malignant glioma therapy. J Shandong Univ Tradi Chin Med. 2010;34:5.

9. Romero-Cordoba SL, Ivan SG, Mauricio RD, Alfredo HM. miRNA biogenesis: biological impact in the development of cancer. Cancer Biol Ther. 2014;15(11):1444-1455. doi:10.4161/15384047.2014.955442 
10. Pichler M, Calin GA. MicroRNAs in cancer: from developmental genes in worms to their clinical application in patients. $\mathrm{Br}$ J Cancer. 2015;113(4):569-573. doi:10.1038/bjc.2015.253

11. Weifeng W, Erdong Z, Changjun L. MicroRNAs in tumor angiogenesis. Life Sci. 2015;136:28-35. doi:10.1016/j.1fs.2015.06.025

12. Kohlhapp FJ, Mitra AK, Lengyel E, Peter ME. MicroRNAs as mediators and communicators between cancer cells and the tumor microenvironment. Oncogene. 2015;34(48):5857-5868. doi:10.1038/ onc. 2015.89

13. Bing Z, Er-Bao B, Jia L, Jun L. New advances of microRNAs in glioma stem cells, with special emphasis on aberrant methylation of microRNAs. J Cell Physiol. 2014;229(9):1141-1147. doi:10.1002/jcp.24540

14. Yang H, Wang Y. Five miRNAs considered as molecular targets for predicting neuroglioma. Tumor Biol. 2015;37(1):1-9.

15. Qiu ZK, Liu N, Zhao SF, et al. MiR-1298 expression correlates with prognosis and inhibits cell proliferation and invasion of gastric cancer. Eur Rev Med Pharmacol Sci. 2018;22(6):1672-1679.

16. Bertolino E, Reimund B, Wildt-Perinic D, Clerc RG. A novel homeobox protein which recognizes a TGT core and functionally interferes with a retinoid-responsive motif. J Biol Chem. 1995;270(52):31178-31188. doi: $10.1074 /$ jbc. 270.52 .31178

17. Wotton D, Lo RS, Lee S, Massagué J. A Smad transcriptional corepressor. Cell. 1999;97(1):29-39. doi:10.1016/S0092-8674(00)80712-6

18. Wotton D, Taniguchi K. Functions of TGIF homeodomain proteins and their roles in normal brain development and holoprosencephaly. Am J Med Genet C Semin Med Genet. 2018;178(5):128-139. doi:10.1002/ajmg.c.31612

19. Pellatt AJ, Mullany LE, Herrick JS, et al. The TGF $\beta$-signaling pathway and colorectal cancer: associations between dysregulated genes and miRNAs. J Transl Med. 2018;16(1):191. doi:10.1186/s12967018-1566-8

20. Yang H, Zhang H, Pan T, Wang H, Wang Y. Benzo(a)pyrene promotes migration, invasion and metastasis of lung adenocarcinoma cells by upregulating TGIF. Toxicol Lett. 2018;294:11-19. doi:10.1016/j.toxlet.2018.05.005

21. Wang Y, Li L, Wang H, Li J, Yang H. Silencing TGIF suppresses migration, invasion and metastasis of MDA-MB-231 human breast cancer cells. Oncol Rep. 2018;39(2):802-808. doi:10.3892/ or.2017.6133
22. Zi-Miao L, Tseng JT, Duang-Yang H, Huei-Sheng H. Suppression of TG-interacting factor sensitizes arsenic trioxide-induced apoptosis in human hepatocellular carcinoma cells. Biochem J. 2011;438(2):349. doi:10.1042/BJ20101653

23. Shaw EJ, Brian H, David H, et al. Gene expression in oligodendroglial tumors. Anal Cell Pathol. 2016;33(2):81-94. doi:10.1155/2010/ 304806

24. Zhaohui L, Yu T, Nan T, et al. Aberrant alternative splicing pattern of ADAR2 downregulates adenosine-to-inosine editing in glioma. Oncol Rep. 2015;33(6):2845-2852. doi:10.3892/or.2015.3907

25. Wang SF, Wu MY, Cai CZ, Li M, Lu JH. Autophagy modulators from traditional Chinese medicine: mechanisms and therapeutic potentials for cancer and neurodegenerative diseases. $J$ Ethnopharmacol. 2016;194:861-876. doi:10.1016/j.jep.2016.10.069

26. Ge X, Gong L. MiR-590-3p suppresses hepatocellular carcinoma growth by targeting TEAD1. Tumour Biol. 2017;39(3):1010428317695947. doi:10.1177/1010428317695947

27. Zhou Y, Dang J, Chang KY, et al. miR-1298 inhibits mutant KRAS-driven tumor growth by repressing FAK and LAMB3. Cancer Res. 2016;76(19):5777. doi:10.1158/0008-5472.CAN-15-2936

28. Wang CM, Cheng BH, Xue QJ, Chen J, Bai B. MiR-1298 affects cell proliferation and apoptosis in C6 cells by targeting SET domain containing 7. Int J Immunopathol Pharmacol. 2017;30(3):039463201772054. doi:10.1177/0394632017720546

29. Wang $\mathrm{Y}$, Wang $\mathrm{H}$, Gao H, et al. Elevated expression of TGIF is involved in lung carcinogenesis. Tumor Biol. 2015;36 (12):9223-9231. doi:10.1007/s13277-015-3615-8

30. Guo X, Yang Y, He W, Wu W. TGIF1 promoted the growth and migration of cancer cells in nonsmall cell lung cancer. Tumor Biol. 2015;36(12):9303-9310. doi:10.1007/s13277-015-3676-8

31. Wang Y, Pan T, Wang H, et al. Silencing of TGIF attenuates the tumorigenicity of A549 cells in vitro and in vivo. Tumor Biol. 2016;37(9):1-6.

32. Carol W, Plessis DG, Du JKA, et al. Molecular pathology and clinical characteristics of oligodendroglial neoplasms. Ann Neurol. 2005;57 (6):855-865. doi:10.1002/ana.20496

\section{Publish your work in this journal}

Cancer Management and Research is an international, peer-reviewed open access journal focusing on cancer research and the optimal use of preventative and integrated treatment interventions to achieve improved outcomes, enhanced survival and quality of life for the cancer patient.
The manuscript management system is completely online and includes a very quick and fair peer-review system, which is all easy to use. Visit http://www.dovepress.com/testimonials.php to read real quotes from published authors. 